\title{
Topography analysis in the NPMM-200
}

\author{
Emiljano Meta ${ }^{1}$, Eberhard Manske ${ }^{1}$ \\ 1 Technische Universität IImenau, Institute of Process Measurement and Sensor Technology, IImenau, \\ Deutschland \\ E-Mail: emiljano.meta@tu-ilmenau.de
}

\begin{abstract}
:
Using a specially developed combination of phase shifting and image processing algorithms will be performed a specific analysis of relatively rough and unknown surfaces (with or without structures). These algorithms are able to evaluate phase-shifted interference images and calculate topographic data from them. Interference images, which are used for the analysis, are acquired with the help of the high-precision nanopositioning and nano-measuring machine (NPMM-200) of the Technical University of Ilmenau [1, 2].
\end{abstract}

Keywords: NPMM-200, Topography, edge detection, phase shifting Algorithms, phase unwrapping

\section{Introduction}

Today, interferometry is one of the most versatile measurement techniques in high-precision non-contact measurement technology. The computer-aided evaluation, which led to interesting industrial measuring methods, helped to achieve a fast and very accurate optical detection of interference fringes. The electronic evaluation of the optical phase of the interference images can increase the sensitivity and measurement accuracy to fractions of the (average) wavelength of the used light source.

The phase shifting method (PSM) is one of the most accurate methods in the topography analysis. Several phase-shifted interference images are necessary for this purpose.

The measuring object is illuminated with a spectral wide light source. Unlike measuring with coherent radiation, it results in a Gaussianshaped profile of the spectral intensity due to the spectral width. This is caused by the superposition of the object and reference wave. The phase-shifted interference images are generated while the object is moved in small equidistant steps relative to the interferometer. These are acquired and saved using a camera microscope. From these images, a three-dimensional topography of a measurement object can be calculated and graphically displayed.

\section{Mechanical setup}

The following components are necessary for the interferometer to generate interference images in the NPMM-200: white light source, Mirau objective, camera microscope and a computer for data acquisition and evaluation.

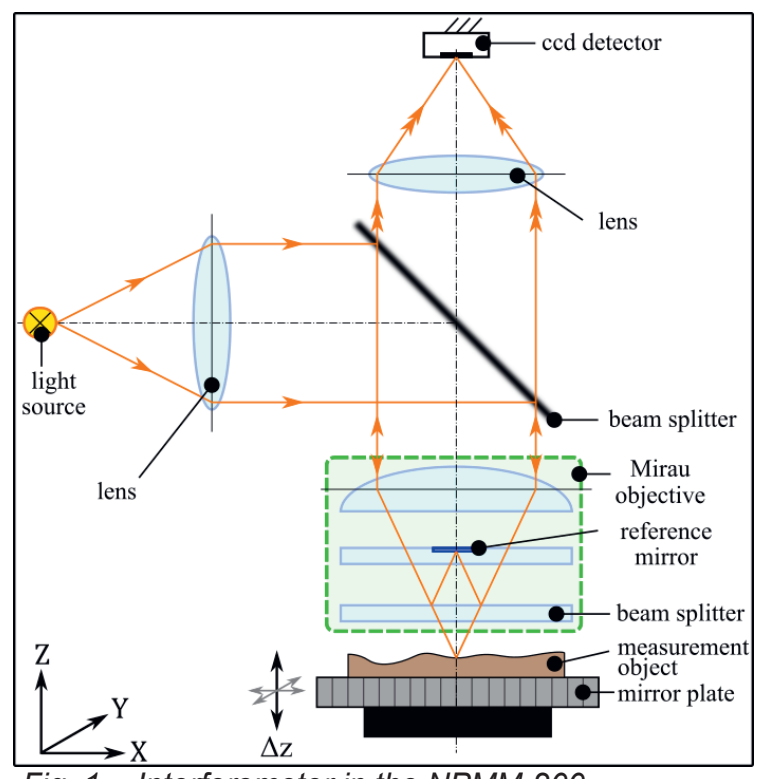

Fig. 1. Interferometer in the NPMM-200

\section{Phase shifting method (PSM)}

This is a dynamic spatial phase shifting method [3]. An interference pattern can be described in the two-beam interference arrangement as follows:

$$
I=I_{0} \cdot\left(1+\gamma_{0} \cdot \cos \left(\phi_{0}+\delta\right)\right)
$$

Here, a phase shift of $\delta=90^{\circ}$ is added between the reference and the measurement object beam, see Figure 2 . This is equivalent to a scanning interval of $\Delta Z=\lambda / 8$, see equation (2). For the solution of equation (1) are at least three phase-shifted interference images necessary. 


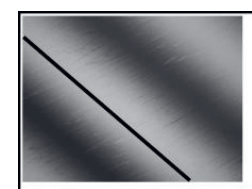

a) $\delta=0$

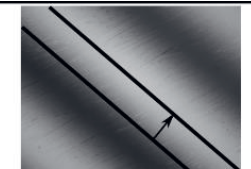

b) $\delta=\pi / 2$

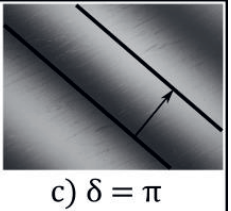

c) $\delta=\pi$
Fig. 2. Phase-shifted interference images

The phase $\varphi_{0}$ contains the information about the optical path difference and the following equation describes the correlation between the two quantities:

$$
\frac{\Delta Z}{\lambda / 2}=\frac{\phi_{0}}{2 \pi} \Rightarrow \Delta Z=\frac{\pi / 2 \cdot \lambda / 2}{2 \pi}=\frac{\lambda}{8}
$$

For the phase analysis is used in this case a so-called 15-step-algorithm, which is relatively robust against small deviations of the phase angle [4]. Therefore, it is necessary to generate (at least) 15 interference images. The more images are used in the analysis, the better will be the result. However, the only disadvantage of this method is that only polished surfaces (e.g. mirror surfaces) can be analysed, which do not contain edges (e.g. in the case of studs). Edges or steps on the measuring object surface lead to phase jumps. If these are higher (lower) than $\pi(-\pi)$, they cannot be clearly identified. In order to overcome this limitation, it will be attempted to detect and extract the different surface areas from the structural surfaces. Special edge detection algorithms are very important.

Furthermore, to avoid discontinuities and to fulfil Nyquist's sampling theorem, an interference fringe should cover at least two pixels on the detector.

\section{Edge detection}

The edge detection algorithms contribute to the detection and automatic selection of the individual surface areas of a structural surface, see figure 3 .

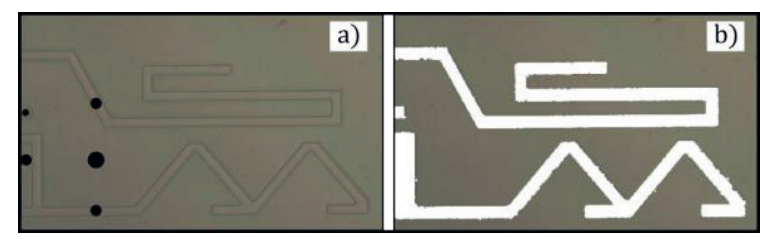

Fig. 3. Edge detection and selection of surface areas

In a first step, only the large area part of figure 3 a) is selected and then evaluated using the PSM algorithm, see figure $3 \mathrm{~b}$ ). Phase jumps occur, which will have to be corrected, see figure 4 a).

All other surface areas that are shown in figure 3 a) (marked with black dots) are saved as objects and then evaluated one by one.

\section{Phase unwrapping}

For relatively complicated structural surfaces, phase jumps can also occur that are quite difficult to unfold, see figure 4 a). For this purpose, there are specially developed algorithms that perform phase unwrapping in two dimensions, see figure $4 \mathrm{~b}$ ) and c).

\section{Results}

The result from the edge detection, phase shifting method and 2D phase unwrapping is shown in the next figure (d)).

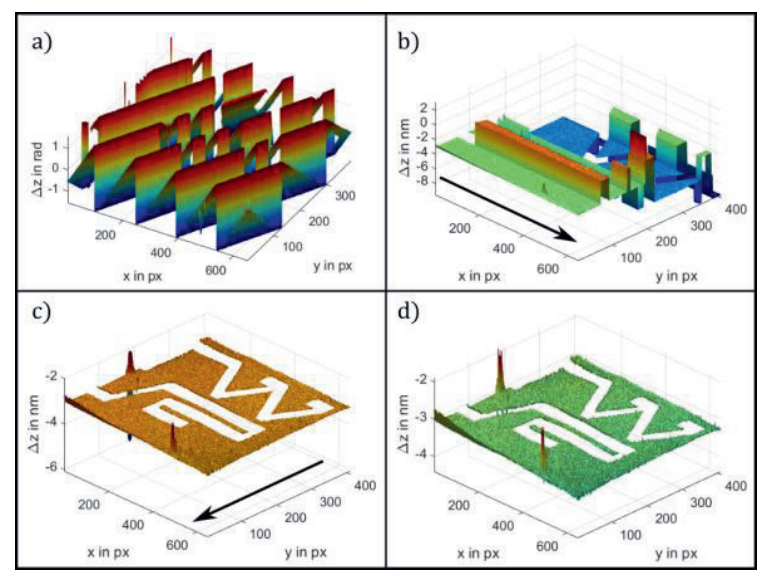

Fig. 4. Phase unwrapping and final result

The height differences of the structure shown above were successfully measured. This has been achieved by direct implementation of the 15-step and 2D phase-unwrapping algorithm without data smoothing, windowing or manual intervention. This would not have been easily possible with conventional phase-unwrapping algorithms.

\section{References}

[1] G Jäger; E Manske; T Hausotte; A Müller; F Balzer, Nanopositioning and nanomeasuring machine NPMM-200-a new powerful tool for largerange micro- and nanotechnology, Surf. Topogr.: Metrol. Prop. (Surface Topography: Metrology and Properties), doi: 10.1088/2051-672X/4/3/034004

[2] NPMM-200, Nanopositionier- und Nanomessmaschine, Technische Universität IImenau, Link: https://www.tu-ilmenau.de/sfb622/transfer-undverbundprojekt-npmm-200/zielstellung/

[3] H.J. Tiziani, Rechnerunterstützte LaserMeßtechnik/ Computer aided laser measurement techniques, tm - Technisches Messen, 221-223; doi: 10.1524/teme.1987.54.6.221

[4] P. D. Groot, 101-frame algorithm for phaseshifting interferometry; doi: 10.1117/12.28 